\title{
The Role of the Jet Emission in Young Radio Sources
}

\author{
Giulia Migliori
}

Harvard-Smithsonian Center for Astrophysics, 60 Garden street Cambridge, MA 02138, USA email: gmigliori@cfa.harvard.edu

\begin{abstract}
We investigated the contribution of the jet to the observed high energy emission in a sample of young and compact radio quasars. For the first time, we compared the Fermi-LAT and Chandra observations of the sample to $\gamma$-ray and X-ray luminosities predicted assuming a jet synchrotron and inverse Compton radiative model. The simulations performed for a reasonable set of model parameters and assumptions provide constraints on the minimum jet power $\left(L_{j e t, k i n} / L_{d i s k}>0.01\right)$, on the contribution of the jet to the X-ray emission, and on the particles to magnetic field energy density ratios.
\end{abstract}

Keywords. galaxies: active, galaxies: jets, gamma-rays: galaxies

\section{Introduction}

Giga-Hertz Peaked Spectrum (GPS) and Compact Steep Spectrum (CSS) sources are powerful and compact radio sources, with typical convex radio spectra characterized by turn-over frequencies in the GHz-MHz band (O'Dea 1998 for a review). Their radio morphology shows lobes, hot spots and jets as for the classical radio sources, but scaled down to galactic or even smaller dimensions. Their spectrally and dynamically estimated ages support the hypothesis that they are the first stage in the evolution of extragalactic radio sources. The main questions on young radio sources concern their evolution and interaction with the environment, i.e. the interstellar medium (ISM) and host galaxy.

The high-energy domain may provide important clues on the most energetic processes associated with the first phase of the source expansion (see e.g. Wagner et al. 2012). $X M M$-Newton and Chandra observations have confirmed that these sources are X-ray emitters $\left(L_{X} \sim 10^{42}-10^{46} \mathrm{erg} \mathrm{s}^{-1}\right.$, Siemiginowska et al. 2008, Tengstrand et al. 2009). However, due to observational limits, the nature of their X-ray emission is still under debate, and it is difficult to estimate the non-thermal emission of the jet and lobes and the one related to the accretion processes (either the X-ray thermal emission of the innermost part of the disk or that resulting from Comptonization of disk photons by electrons in a hot corona).

The $\gamma$-ray band can be useful to study many aspects of the physics of young radio sources. At these frequencies the disk-corona component is expected to rapidly drop in intensity, while both beamed and unbeamed non-thermal emission could be still strong and detectable in powerful sources (see e.g. Stawarz et al. 2008). So far a systematic investigation has not yet been performed with Fermi-LAT. Here, we present the results of our $\gamma$-ray study on a sample of young radio quasars detected in the X-ray band with Chandra. The main goals are to look for $\gamma$-ray detections of young radio sources, and to constrain the entire non-thermal high-energy emission of these objects. 


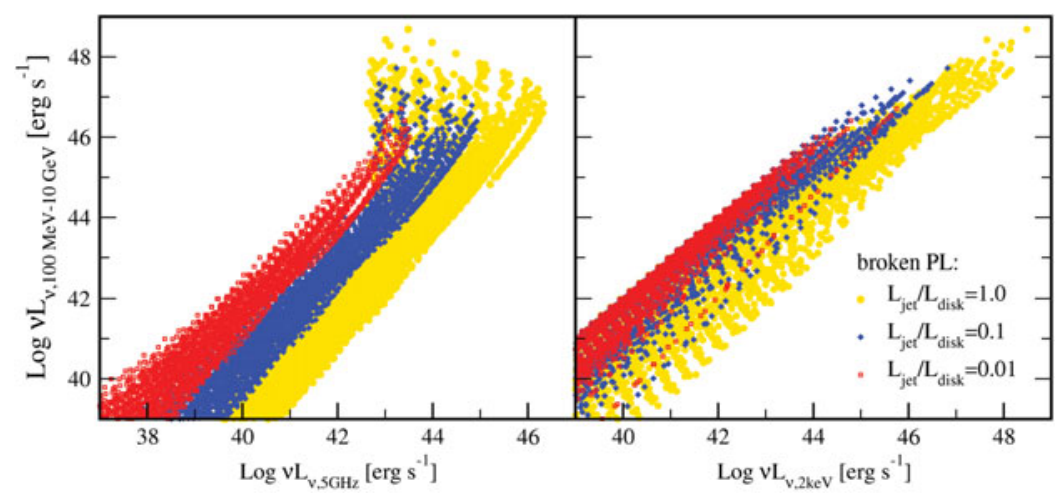

Figure 1. Gamma-ray (100 MeV - $10 \mathrm{GeV})$ luminosities are plotted as a function of the radio ( $5 \mathrm{GHz}$, left panel) and X-ray (2 keV, right panel) luminosities for the simulated jets.

\section{Modeled Jet Emission}

Our approach consists of a comparison between model predictions and data. The broadband jet emission is calculated using a leptonic radiative model. In this way, we generate a library of broad-band spectral energy distributions (SED) for a wide range of jet parameters. Then, we compare the simulated luminosity distributions in the radio, X-ray and $\gamma$-ray regimes with observations for a sample of GPS and CSS quasars.

In the model, the bulk of the jet emission is produced in a spherical region with radius $R$, located at a distance, $z_{d}$, from the central black hole. At low frequencies, the jet radiates via synchrotron mechanism. The high energy emission is produced via Compton scattering. Seed photons for the inverse Compton (IC) mechanism are the synchrotron emission of the knot and external radiation fields (synchrotron-self-Compton, SSC, and external Compton, EC, processes, respectively). External photons include the UV disk photons, the IR emission from the surrounding dust and synchrotron emission produced in a second region located at the base of the jet (see Migliori et al. 2012). The details of the model are illustrated in Migliori et al. (2014).

In the simulations, the jet linear size, $L S$, the bolometric disk luminosities, $L_{d i s k}$, the bulk motion of the emitting region, $\Gamma$, and the observer viewing angle $\theta$ are free input parameters, while $z_{d}$ is set equal to $L S, R$ is $0.1 L S$, and the dust emission $L_{d u s t}$ is proportional to $L_{d i s k}$. The shape of the radiating electrons' energy distribution (EED) is either a simple power-law or a broken power-law $\dagger$. We linked together the jet and disk powers, with their ratio, $L_{j e t, k i n} / L_{d i s k}$, spanning between 0.01 and 1 . We assumed a moderate dominance of particles over magnetic field energy density in the knot: $U_{e}^{\prime} / U_{B}^{\prime}=10\left(U_{e}^{\prime}\right.$ is the energy density of the radiating electrons and $U_{B}^{\prime}$ the magnetic field energy density).

Our simulations cover the range of LS and $L_{\text {disk }}$ values of the sample of GPS and CSS quasars in Siemiginowska et al. (2008): $L S=0.01-10 \mathrm{kpc}$ and $L_{\text {disk }}=10^{45}-10^{47} \mathrm{erg}$ $\mathrm{s}^{-1}$ respectively. Values of $\Gamma$ and $\theta$ in the range $1.4-10$ and $10^{\circ}-50^{\circ}$ were assumed. In Fig. 1 , we show the $\gamma$-ray luminosities in the $0.1-10 \mathrm{GeV}$ band $\left(L_{100 \mathrm{MeV}-10 \mathrm{GeV}}\right)$ of the simulated sources as a function of their radio $\left(L_{5 G H z}\right.$, left panel) and X-ray ( $L_{2 k e V}$, right panel) luminosities at $5 \mathrm{GHz}$ and $2 \mathrm{keV}$ respectively. For this example the EED is a broken powerlaw and the luminosities are in the observer reference frame. We obtained large $\gamma$-ray luminosities: sources with $L_{5 G H z} \gtrsim 10^{43} \mathrm{erg} \mathrm{s}^{-1}$ can reach $L_{100 \mathrm{MeV}-10 \mathrm{GeV}} \gtrsim 10^{44} \mathrm{erg} \mathrm{s}^{-1}$.

$\dagger$ The minimum and maximum electron Lorentz factors, $\gamma_{\min }$ and $\gamma_{\max }$, are 10 and $10^{5}$, respectively, the spectral index of the single power law is $\sim 2.7$, and the spectral indexes and the energy break of the broken power law are 2.1, 2.4 and $\sim 2 \times 10^{3}$, respectively. 


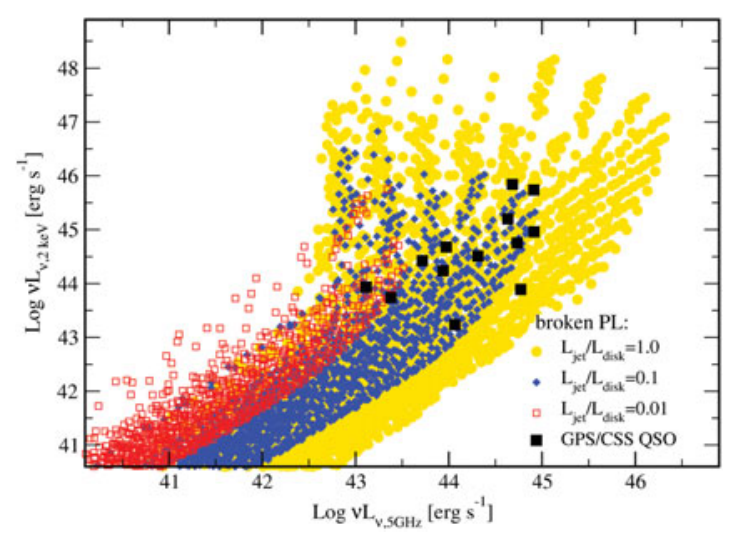

Figure 2. X-ray luminosities $L_{2 \mathrm{keV}}$ of the sample of young radio quasars and the simulated sources as a function of radio luminosities $L_{5 G H z}$ (observer rest frame).

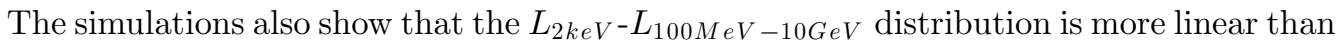
the $L_{5 \mathrm{GH} z}-L_{100 \mathrm{MeV}-10 \mathrm{GeV}}$ distribution, in particular when $L_{5 \mathrm{GH}} \gtrsim 10^{43} \mathrm{erg} \mathrm{s}^{-1}$. Thus, in order to select sources for a $\gamma$-ray detection both radio and X-ray observations are important.

\section{Sample and Fermi-LAT data}

For a comparison with observations, we selected a sample of 13 GPS and CSS quasars. All the sources have radio and X-ray Chandra observations (see Siemiginowska et al. 2008). We looked for $\gamma$-ray detections and analyzed the Fermi-LAT data collected during 46 months of operation (August 2008 to February 2012). There was no statistically significant $\gamma$-ray detection of the young radio sources in our sample for the considered Fermi-LAT data set. The only source detected with high significance (TS $=514$ ) is PKS1127-14. However, the classification of this source has been revised from a young radio source to a Flat Spectrum Radio Quasar. For all the other sources we derived the LAT upper limits (red triangles in Fig 3, left panel).

\section{Results}

The comparison between the simulated luminosities and multi-band data for the observed sample allowed us to place constraints on the jet power and composition and on its contribution to the high-energy emission.

Jet to Disk Power Ratio: in Fig. 2, we plot the $L_{2 k e V}$ of the quasars in the sample and of the simulated sources as a function of their $L_{5 G H z}$. The quasars occupy the highluminosity region of the $L_{5 G H z}-L_{2 k e V}$ plot. This is well-sampled by our simulations when $L_{j e t, k i n} / L_{\text {disk }}=1$ (yellow circles), while the overlap with the $L_{j e t, k i n} / L_{\text {disk }}=0.01$ distribution (red empty squares) is only marginal. Note that this result mainly depends on the radio luminosities and that an overestimate of the jet contribution to the observed X-ray emission would make the discrepancy between data and simulations (when $L_{\text {jet }, \text { kin }} / L_{\text {disk }}<1$ ) even larger. Thus, a $L_{\text {jet,kin }} / L_{\text {disk }}>0.01$ ratio seems required for the range of disk luminosities of the GPS and CSS sources in the observed sample.

Gamma-ray Emission: in Fig. 3 (left panel), the Fermi-LAT upper limits and the sim-

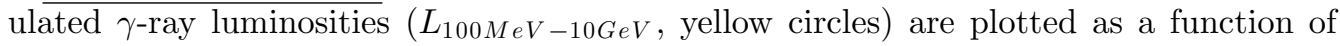



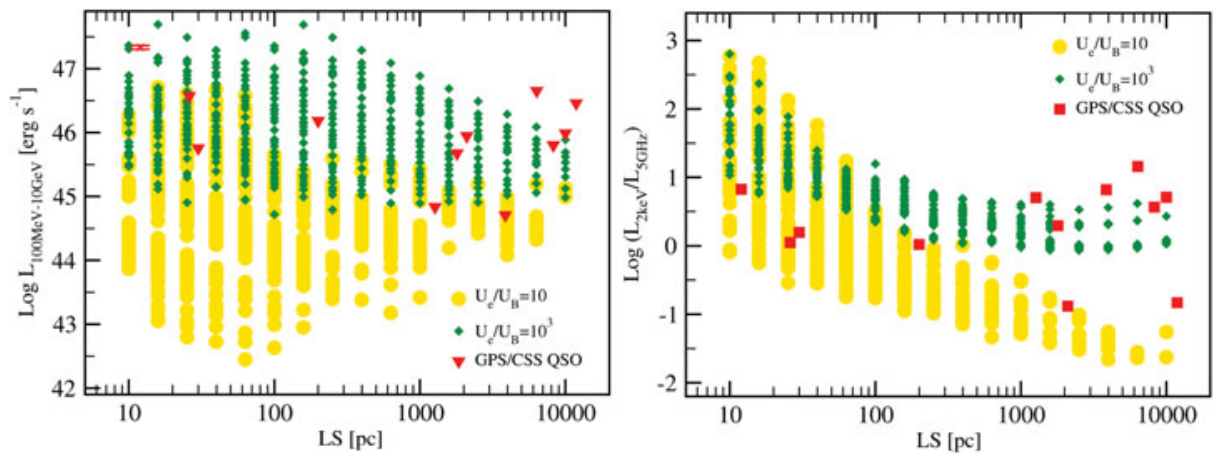

Figure 3. Left panel: simulated $100 \mathrm{MeV}-10 \mathrm{GeV}$ luminosities and Fermi-LAT results are plotted as a function of the linear size, LS. Right panel: comparison of the $2 \mathrm{keV}$ to $5 \mathrm{GHz}$ luminosity ratio as a function of the linear size LS for the simulated distributions and the sample of young radio quasars.

the linear size, $L S \dagger$. For this comparison, a subsample was selected from the simulated distribution obtained for $L_{j e t, k i n} / L_{d i s k}=1$, in order to match the values of the observables of the sample $\left(10^{43} \leqslant L_{5 G H z} \leqslant 10^{45} \mathrm{erg} \mathrm{s}^{-1}, 10^{43} \leqslant L_{2 k e V} \leqslant 10^{46}\right.$ erg s$^{-1}$ in addition to $10^{45} \leqslant \mathrm{~L}_{\text {bol/disk }} \leqslant 10^{47} \mathrm{erg} \mathrm{s}^{-1}, 10 \mathrm{pc} \leqslant \mathrm{LS} \leqslant 10 \mathrm{kpc}$ ). The model-predicted $\gamma$-ray fluxes are compatible with the derived upper limits, however the LAT sensitivity is the main limit for a test of the jet model at these energies.

Origin of the $X$-ray Emission: we tested the model for the ratio of X-ray to radio luminosity $\left(L_{2 k e V} / L_{5 G H z}\right)$ as a function of LS (see Fig. 3, right panel). The modeled $L_{2 \mathrm{keV}}-L_{5 \mathrm{GH} z}$ ratios (yellow circles) are in good agreement with the ones of the quasar sample (red solid squares) only for $\mathrm{LS} \lesssim 1 \mathrm{kpc}$. For sources with $\mathrm{LS} \gtrsim 1 \mathrm{kpc}$, the model predicts X-ray luminosities lower than the observed ones. We conclude that either the bulk of the jet X-ray emission is always produced at $z_{d} \lesssim 1 \mathrm{kpc}$, or the observed X-ray emission is due to an additional component not related to the jet, such as the disk-corona emission.

Particle to Magnetic Field Energy Densities: we checked the previous result against different model parameter values and assumptions. Large deviations from the energy equipartition between particles and magnetic field $\left(U_{e}^{\prime} / U_{B}^{\prime} \gtrsim 10^{3}\right)$ are required to produce the observed X-ray luminosities (green diamonds in right panel of Fig. 3). However, for the majority of the sources this would imply $\gamma$-ray fluxes detectable by Fermi -LAT (see left panel in Fig. 3). This suggests that $U_{e}^{\prime} / U_{B}^{\prime}$ in the jets of young radio sources are in broad agreement with energy equipartition assumption.

\section{References}

Kataoka, J. \& Stawarz, Ł. 2005, ApJ, 622, 797

Migliori, G., et al. 2012, ApJ, 749, 107

Migliori, G., et al. 2014, ApJ, 780, 165

O'Dea, C. P. 1998, PSP, 110, 493

Siemiginowska, A., et al. 2008, ApJ, 684, 811

Stawarz, Ł., et al. 2008, ApJ, 680, 911

Tengstrand, O., et al. 2009, A\&SA, 501, 89

Wagner, A. Y., et al. 2012, ApJ, 757, 136

$\dagger$ One source, for which the LS measure was missing, was excluded for this analysis. 